\title{
SEA ICE DRIFT MONITORING IN THE BOHAI SEA BASED ON GF4 SATELLITE
}

\author{
Zhao Yingjie*, Wei Pan, Zhu Hui, Xing Baiyang \\ Shandong University of Science and Technology, Qingdao 266590, China. 1124525821@qq.com
}

KEY WORDS: GF4 image, Sea ice, Sift, MCC, Monitor

\begin{abstract}
:
The Bohai Sea is the inland sea with the highest latitude in China. In winter, the phenomenon of freezing occurs in the Bohai Sea due to frequent cold wave influx. According to historical records, there have been three serious ice packs in the Bohai Sea in the past 50 years which caused heavy losses to our economy. Therefore, it is of great significance to monitor the drift of sea ice and sea ice in the Bohai Sea. The GF4 image has the advantages of short imaging time and high spatial resolution. Based on the GF4 satellite images, the three methods of SIFT (Scale invariant feature - the transform and Scale invariant feature transform), MCC (maximum cross-correlation method) and sift combined with MCC are used to monitor sea ice drift and calculate the speed and direction of sea ice drift, the three calculation results are compared and analyzed by using expert interpretation and historical statistical data to carry out remote sensing monitoring of sea ice drift results. The experimental results show that the experimental results of the three methods are in accordance with expert interpretation and historical statistics. Therefore, the GF4 remote sensing satellite images have the ability to monitor sea ice drift and can be used for drift monitoring of sea ice in the Bohai Sea.
\end{abstract}

\section{INTRODUTION}

The Bohai Sea is located in $37^{\circ} 07^{\prime}-41^{\circ} 00$ 'N, $117^{\circ} 35^{\prime}-$ $121^{\circ} 10^{\prime} \mathrm{E}$ (figure 1), which is the semi-enclosed sea of our country. The economic development of the Bohai rim region, the heavy sea traffic, the exploration and development of offshore oil and gas are in the ascends. However, in the winter each year, human activities such as the development of marine resources, the engineering design of harbors and coasts, and the transportation of seas are all affected to different degrees due to the formation, movement, and changes of sea ice. In particular, serious ice conditions have great harm to offshore oil platforms and other offshore structures, hinder economic construction and development in the region, and affect the normal activities of warships and submarines in the region. Therefore, it is very important to monitor sea ice in Bohai Sea area. In recent decades, there have been many serious sea ice disasters in the Bohai Sea of China, which have caused huge losses to China's economy. From February to March 1969, the Bohai Sea suffered a particularly large ice seal, and the Bohai Sea was almost completely frozen. The maximum thickness was $100 \mathrm{~cm}$, resulting in the destruction of multiple ships and the collapse of the oil platform, and the fishermen could not go to sea. In the winter of 2009 2010, an immense sea ice disaster occurred in the Bohai Sea, and the loss of marine fishery in Shandong and Liaoning provinces exceeded 3 billion RMB. Even

${ }^{*}$ Corresponding author 
in light ice years, sea ice will still bring disasters, such as the drift of sea ice, which will cause vibration in offshore buildings, causes destruction of buildings, and will not work properly.

The Bohai Sea ice belongs to one year ice, and the development of sea ice can be divided into three stages, ie, the initial ice period, frozen period, and final ice age. At the beginning of period is from the beginning to the frozen ice day, this time is the process of sea ice

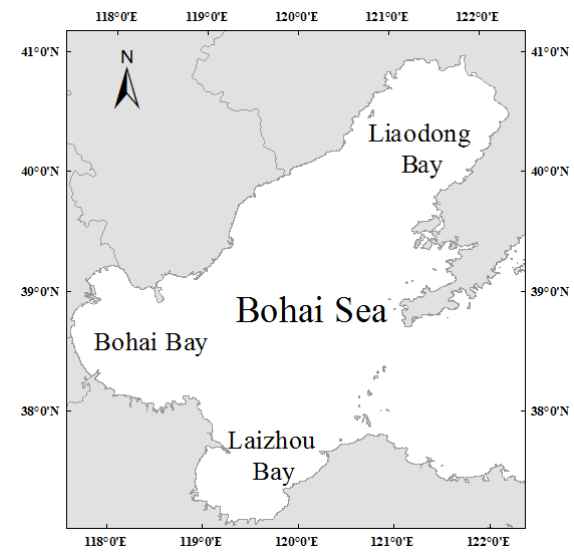

(a) growing. The freezing period refers to thaw frozen day, this time the ice is serious, ice concentration is greater than $70 \%$, so the frozen day is called the most serious ice age. The final glacial period refers to the defrosting day to the final ice day, which changes with the rise of the temperature and the increase of the sea temperature. This paper is mainly based on GF4 satellite images to monitor the drift of Bohai sea ice, which is beneficial to sea ice recognition because of its high spatial resolution $(50 \mathrm{~m})$

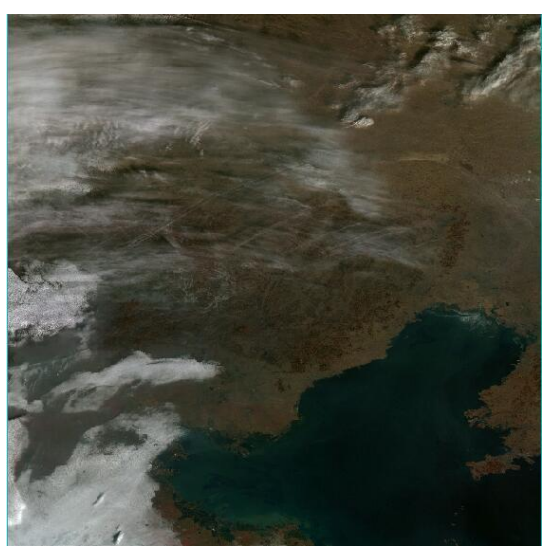

(b)

Figure 1: The study area for this study (a) and the GF4 image (Red: B3, Green: B4, and Blue: B5) (b)

\section{DATA PROCESSING}

\subsection{GF-4 Geostationary Satellite}

Gaofen 4 is a geostationary Earth observation satellite. Unlike most Earth observation satellites which all operate in highly inclined polar orbits, Gaofen 4 operates from geostationary Earth orbit (GEO), which allows uninterrupted coverage of the same area on the ground 24 hours a day. Gaofen 4's imaging payload, developed by Beijing Institute of Space Machinery \& Electronics (508 Institute), consists of two staring optical imagers, one operating in visible light and one in infrared, sharing a common optical system. The spatial resolution is $50 \mathrm{~m}$ for the visible light imager and $400 \mathrm{~m}$ for the infrared imager. Operating from GEO, the imaging package allows the observation of China and its surrounding areas within an area of 7,000 $\mathrm{km} \times 7,000 \mathrm{~km}$, with each individual frame covering

\begin{tabular}{l|c|c|c|c|c}
\hline \multirow{2}{*}{} & Band & $\begin{array}{c}\text { Spectral Range } \\
(\mu \mathrm{m})\end{array}$ & $\begin{array}{c}\text { Spatial } \\
\text { Resolution }(\mathrm{m})\end{array}$ & $\begin{array}{c}\text { Swath } \\
(\mathrm{m})\end{array}$ & Revisit Time (s) \\
\hline \multirow{3}{*}{ VNIR } & 1 & $0.45 \sim 0.90$ & & & \\
\cline { 2 - 3 } & 2 & $0.45 \sim 0.52$ & & \multirow{2}{*}{50} & 20 \\
\cline { 2 - 3 } & 4 & $0.52 \sim 0.60$ & $0.63 \sim 0.69$ & & \\
\cline { 2 - 4 } & 5 & $0.76 \sim 0.90$ & & & \\
\hline MWIR & 6 & $3.5 \sim 4.1$ & 400 & & \\
\hline
\end{tabular}

VNIR (Visible and Near Infrared), MWIR (Middle Wave Infrared)

Table 1. GF-4 Satellite payload technical specification 
an area of $400 \mathrm{~km} \mathrm{x} 400 \mathrm{~km}$, and a capacity for minutelevel temporal resolution monitoring. The satellite was developed by China Academy of Space Technology (CAST), based on their new GEO remote-sensing satellite bus. It has orbital mass of 4,600 kg, and was designed for a life span of 8 years. The satellite was placed into orbit by a CZ-3B launch vehicle from the Xichang Satellite Launch Centre on 29 December 2015 After four orbital manoeuvres, the satellite was positioned at $105.6^{\circ} \mathrm{E}$ on GEO on 4 January 2016.

The shape of the reflection spectrum curve of annual sea ice in the Bohai Sea is similar to that of seawater and snow. The reflection peak level is higher than seawater, but much lower than snow, and has many feature points. As can be seen from the overall shape of the figure below, from the visible to the near-infrared, the reflectivity of snow is up to $80 \%$, followed by ice (between $30 \%$ and $60 \%$ ), and the reflectivity of water is the lowest (10\%), to infrared wavelengths with a wavelength equal to or greater than $1200 \mathrm{~nm}$, the reflectivity of ice and water is close to the total absorber and difficult to distinguish. GaoFen4 band wavelengths are shown in the above table, therefore, GF4 satellite remote sensing images are suitable for sea ice monitoring research.

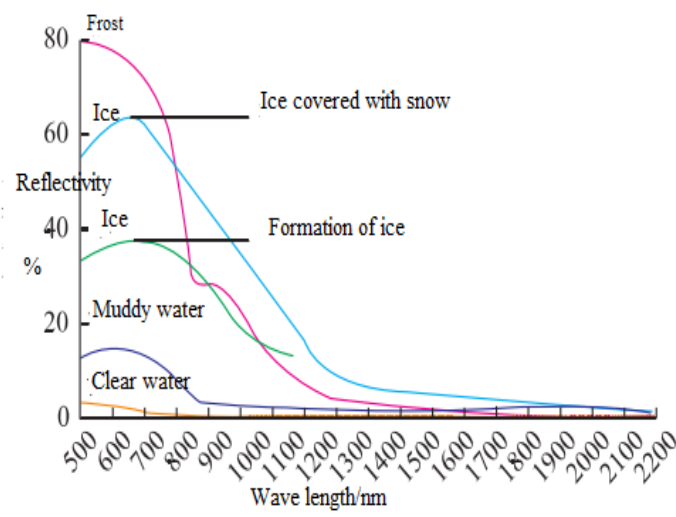

Fig. 2. Comparison of spectral features of snow, sea ice and sea water. From the literature (Ding Dewen, 2000)

\subsection{Data Processing}

Since the distributed high-resolution 4 remote sensing satellite imagery is a L1A product and cannot be directly used for experiments, we need to do orthographic correction and registration processing for images. Orthographical correction is usually by selecting some ground control points on the picture, and use the original has obtained the pictures within the scope of the digital elevation model (DEM) data, to tilt correction and projection images at the same time correction, image resampling into orthogonal projection image. After the image is directly orthorectified, we find that there are large positional deviations in the images of the same region at different times, the processing done here is to make the images in relative registration. Relative registration refers to selecting an image in the multiple images as the reference image is registered with other related images and its coordinate system is arbitrary.

If the captured satellite images are closer to the coast, the satellite images generally contain land; There may be islands in the sea area far from the coast, and these strong reflectors can create errors in the monitoring of sea ice drift. Therefore, it is necessary to mask the land and islands before monitoring the sea ice drift. The conventional mask method for land and island is to manually extract the coastline from satellite images. The GF-4 remote sensing satellite images, which are used to monitor sea ice drift, include land, sea and sea ice, as well as islands, so satellite images are needed to cover land and island. This paper uses ENVI (the Environment for Visualizing Images) to capture interest in seawater areas and mask land and islands by interest. As shown in the picture, the satellite image of 10:31 on January 6,2017 and 11:31 on January 10, 2017 (Beijing time): 

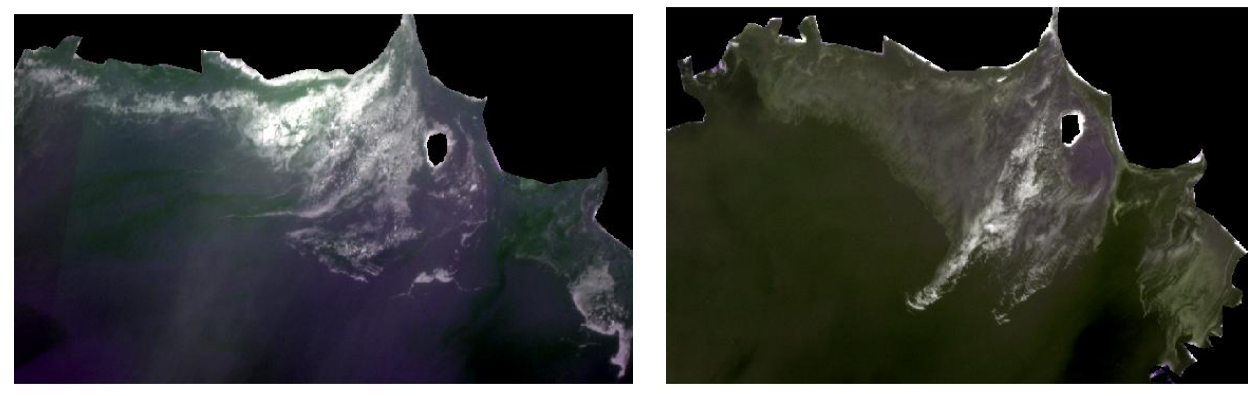

Figure 3: Land and island mask

\section{METHODOLOGY}

\subsection{MCC (maximum cross-correlation method)}

The algorithm principle of the maximum cross correlation method: By calculating the statistical feature values of the image to be matched and the reference image, the best matching area in the two images is obtained, the basic idea is to use the size of the mutual relationship between the two images to measure their degree of matching. As shown in the following figure, in two consecutive images, the feature region in the reference image is represented by $f(x, y)$, and the feature region traversing the search reference image in the image to be matched is represented by $g(x, y)$, calculate the correlation coefficient of $f(x, y)$ and $\mathrm{g}(\mathrm{x}, \mathrm{y})$, and take the maximum correlation coefficient as the best matching region, the calculation method is shown in formula (1):

$$
\rho(x, y)=\frac{\operatorname{cov}(f(x, y), g(x, y))}{\sqrt{D_{x, y} D}}
$$

Where $\rho(x, y)=$ correlation coefficient

$$
\begin{aligned}
& D_{x, y}=\text { the variance of } \mathrm{f}(\mathrm{x}, \mathrm{y}) \\
& D=\text { the variance of } \mathrm{g}(\mathrm{x}, \mathrm{y})
\end{aligned}
$$

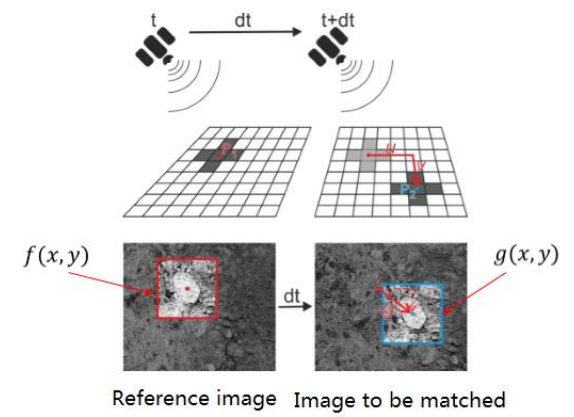

Fig. 4 Illustration of the MCC method. The rectangle with the red outline in the first image is the manually selected feature and the rectangle with the blue outline in the second image is the desired feature.

Assume that two sequential images (reference image and image to be matched) are acquired at different time points $t$ and $t+d t$ respectively, as shown in Fig. 4. For each selected typical feature located at point $P_{1}\left(x_{1}, y_{1}\right)$ in the reference image, the algorithm searches for the best match located at point $P_{2}\left(x_{2}, y_{2}\right)$ in the reference image. The best match is found by computing the cross-correlation between the surface feature of the first image and those of the same size within the search area in the second image, and then choosing the position with the highest crosscorrelation value. According to the position shift in the two images, the drift speed (v) and direction $(\theta)$ can be calculated as follows:

$$
\begin{gathered}
d_{x}=x_{2}-x_{1}, d_{y}=y_{2}-y_{1} \\
v=\sqrt{d_{x}^{2}+d_{y}^{2}} / d t \\
\theta=\arctan \left(d_{y} / d_{x}\right)
\end{gathered}
$$

where $\left(x_{1}, y_{1}\right)$ and $\left(x_{2}, y_{2}\right)=$ image coordinates $\mathrm{v}=$ the sea ice drift speed (in $\mathrm{m} / \mathrm{s}$ )

$d_{x}=$ horizontal displacement

$d_{y}=$ vertical displacement

$d t=$ the time lag between the two images.

\subsection{SIFT (Scale invariant feature transform)}

Scale invariant feature transform, The Sift feature is based on the localized interest points on the object and has nothing to do with the size and rotation of the image, and the tolerance for changes in the light and noise angles is also quite high. The essence of SIFT algorithm can be attributed to the problem of finding feature points (key points) in different scale spaces. 


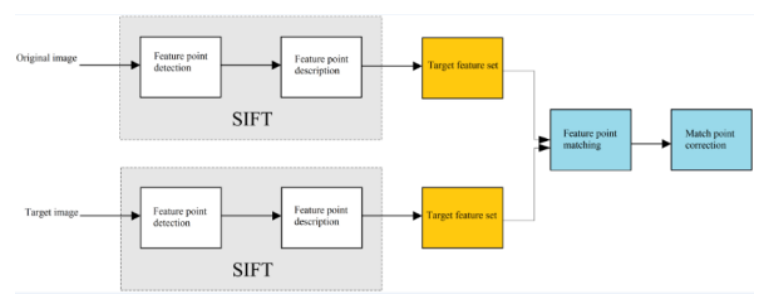

Fig. 5 SIFT flow chart

There are three main processes for object recognition by SIFT algorithm, 1. Extract key points; 2. Adding detailed information (local features) to key points is also known as a descriptor; 3. By comparing the two pairs of feature points (the key points with the feature vectors), we can find a few pairs of feature points matching each other, and the corresponding relationship between the objects is established. Sift calculates the speed and direction of sea ice drift similar to that of MCC, the difference is that Sift calculates the movement speed of a certain point, while MCC calculates the flow velocity of a small area.

\subsection{SIFT and MCC combined algorithm}

The maximum cross-correlation method is to manually extract feature areas, depending on the target interpretation of the person, there will be less or missed selection, manual selection of feature areas is guaranteed but the efficiency will be reduced, so the research methods of extracting feature area automatically. The essence of the sift algorithm is to find key points (feature points) in different scale spaces and calculate the direction of the key points, the key points of SIFT find that there are some very prominent, not because of illumination, affine transformation and noise and other factors change, such as corner points and edge points, dark area bright and bright areas of the dark etc.. Therefore, we combine both MCC and SIFT, first select the feature points with sift, remove the repeated or distant points through cluster analysis, and use the remaining feature points as the center to draw the feature area, and then we use the maximum cross correlation algorithm to traverse the second images, find the corresponding feature matching area, and calculate the sea ice drift speed and direction. The combination of the two algorithms can get more uniform sea ice drift distribution.

The matching of feature areas depends to some extent on the setting of the window size and the selection of the number of features. The following sections discuss the selection of feature window sizes: Feature window size selection must satisfy two conditions: First, the window size must be small enough to ensure that the sea ice movement within the window is uniform, that is, the velocity and direction of movement between adjacent sea ice samples within the window are relatively consistent; The second window size must be large enough to ensure that the window contains enough information for sea ice drift tracking. Based on the above two rules, experiments were performed using $25 * 25,31 * 31$, and $41 * 41$ windows respectively. The experimental results show that the $31 * 31$ window is favorable for the drift tracking of sea ice in the Bohai Sea.

\section{RESULT AND ANALYSIS}

This article is based on the above three methods for GF-4 satellite imagery experiment, $\mathrm{t}$ using data for two images of January 6,2017 , the time interval is four hours, two images of January 11,2018 , the time interval is five hours, and two images of February 6, 2018 time interval is five hours. In different time and different angles, for the same area or location of satellite imaging, the light may be different, which leads to the same pixel area or position in the satellite images may not be the same, cause the error to sea ice monitoring, Therefore, in this paper, two consecutive images are matched by histograms to ensure that the histograms of the two images are similar or identical and reduce the matching error. For some features of ice is not particularly clear images, need to do image enhancement, image purposefully stressed global or local characteristics of image, the original is not clear image clarity or to emphasize some characteristic of interest, to expand the difference between different object in the image features, not interested in inhibition of characteristics, to improve image quality, abundant information, strengthen the image recognition effect. 


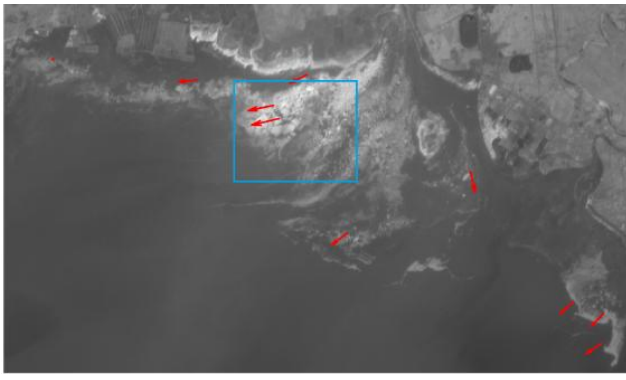

(a)

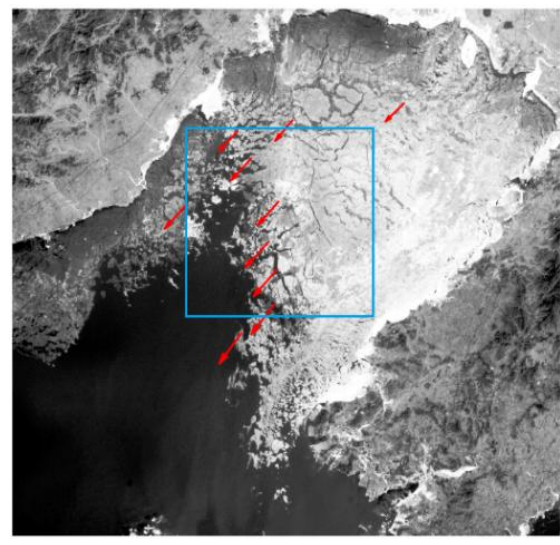

(c)

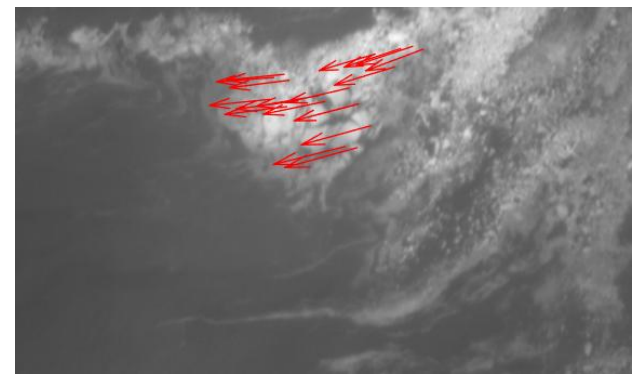

(b)

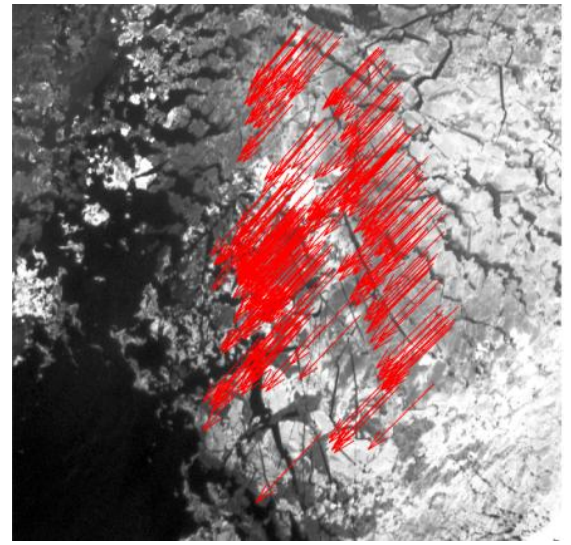

(d)

Fig. 6 (a) (c) Sea ice monitoring based on MCC for 2017/1/06 and 2018/2/06 respectively,

(b)(d) Sea ice monitoring based on sift in the blue area of the two images, respectively.

Drifting monitoring of sea ice in Bohai based on MCC, the extraction of feature area rely on artificial interpretation, to extend the time of selection, where the feature is not particularly easy to leak, optional feature area will be too little, and although the accuracy is high, but the sea ice drift displacement field is evacuated. Based on SIFT method, after filtering error points matching, the remaining drift points are too dense, which is not conducive to obtaining the sea ice drift of the whole region.

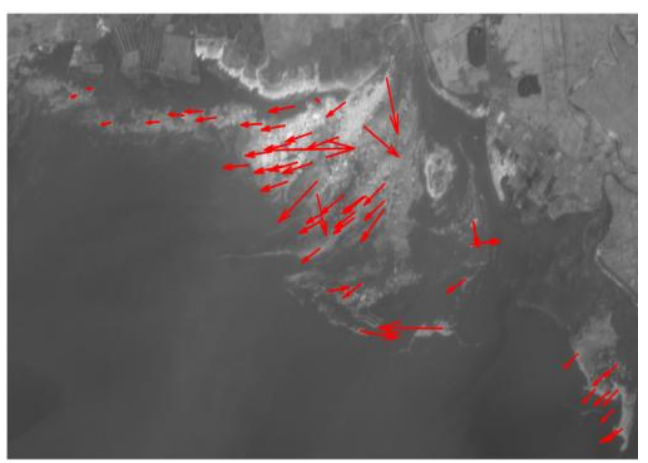

(a)

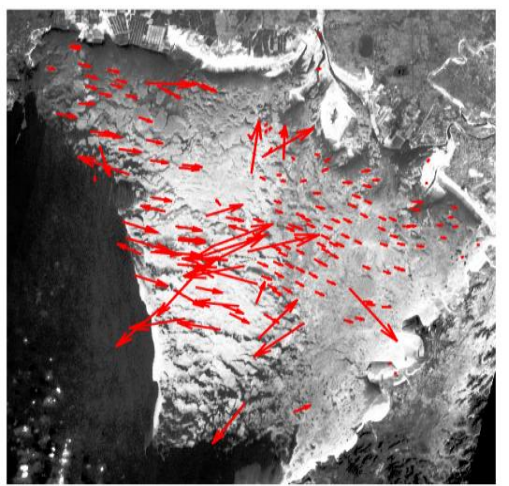

(b)

Fig. 7 (a) (b) the sea ice drift monitoring based on improved algorithm for 2017/1/06 and 2018/1/11 respectively.

Comparing the above two methods, a combination of these two algorithms are more likely to obtain a more uniform distribution of sea ice drift, but also has individual characteristics area matching error, because the image interval time is a bit big, part of the sea ice deformation is large, there are some errors, through expert interpretation and history data validation, the combination of accuracy is about eighty percent. By 
statistical analysis, the drift velocity of sea ice in the experimental results is $0.2 \sim 0.6 \mathrm{~m} / \mathrm{s}$, and the drift of sea ice in some regions reaches $1 \mathrm{~m} / \mathrm{s}$.According to the statistics from November 1963 to March 1978, the average ice drift speed is $0.2-0.4 \mathrm{~m} / \mathrm{s}$. In the vicinity of the eastern coast of northern Liaodong Bay (in the case of the squid ring), the average ice drift speed is about $0.4-0.5 \mathrm{~m} / \mathrm{s}$; the maximum is $0.7-0.9 \mathrm{~m} / \mathrm{s}$, and the maximum drift rate in some areas even exceeds $1.0 \mathrm{~m} / \mathrm{s}$. Liaodong Bay the average speed near the north west coast is $0.3-0.4 \mathrm{~m} / \mathrm{s}$, and the average drift speed in the sea near Qinhuangdao is only $0.2 \mathrm{~m} / \mathrm{s}$. It is the smallest average speed in all sea areas. This is mainly due to the small current velocity in the sea area. In Bohai Bay, the average speed in all directions is relatively close, both being $0.3 \mathrm{~m} / \mathrm{s}$. The maximum ice speed in each direction is quite different, mostly 0.4 to $0.6 \mathrm{~m} / \mathrm{s}$, and individual up to $1.1 \mathrm{~m} / \mathrm{s}$. Laizhou Bay, taking the waters near Longkou as an example, the average ice speed is mostly $0.2-0.4 \mathrm{~m} / \mathrm{s}$, the maximum is $0.4-0.6 \mathrm{~m} / \mathrm{s}$, and the individual is $1.0 \mathrm{~m} / \mathrm{s}$. Take the sea near Dalu Island as an example, average ice the speed is mostly 0.2 to $0.3 \mathrm{~m} / \mathrm{s}$, and the maximum is 0.3 to $0.7 \mathrm{~m} / \mathrm{s}$, and individual up to $1.1 \mathrm{~m} / \mathrm{s}$.

\section{CONCLUSION}

This article combines visual interpretation with historical data to analyze sea ice drift, MCC and SIFT the combination of these two algorithms to monitor sea ice floats, compared with single use two methods have better access to the whole sea ice drift tendency, the algorithm also has certain defects, because the method of MCC searches for the best matching area by traversing second images., calculation efficiency is low.

\section{REFERENCE}

Boroş, E., Roşca, G., Iftene, A., 2009. Using SIFT Method for Global Topological Localization for Indoor Environments. Multilingual Information Access Evaluation II. Multimedia Experiments. Springer Berlin Heidelberg, pp. 277-282.
Bai, S., Liu, Q., Li, H., Wu, H., 1999. Sea ice in the bohai sea of china. Marine Forecasts.

Ding D. W., 2009. Introduction to Engineering Ice Science, Beijing: Ocean Publishing House.

Deng, Z., Chi, T. H., Zhang, X. 2011. Comparative study of multi-method sea ice information extraction based on HJ satellite images. Journal of Minjiang University, 32(2), pp. 122-125.

Lang, W. H., and Meng, J., 2014. Sea ice drift tracking in the Bohai sea using geostationary ocean color imagery, Journal of Applied Remote Sensing, 8(1), 083650 .

W. Yi, S. Huang, F. Wang, 2016.GF-4 played a crucial role in forest conflagrations monitoring, Satellite Appl., vol. 5, no. 5, pp. 49-51 